\title{
The Impact of Size and Inhomogeneity on Power Transmission Network Complex System Dynamics
}

\author{
B. A. Carreras \\ BACV Solutions, \\ Oak Ridge, TN 37831 \\ bacarreras@gmail.com
}

\author{
D. E. Newman \\ Physics Department \\ University of Alaska \\ Fairbanks, AK 99775 \\ denewman@alaska.edu
}

\author{
I. Dobson \\ ECE Department \\ Univ. of Wisconsin \\ Madison, WI 53706 \\ dobson@engr.wisc.edu
}

\begin{abstract}
With ever growing networks in critical infrastructure systems, the impact of network characteristics such as size and homogeneity on their complex system dynamics and risk of failure becomes crucial. In this paper we investigate the effect of highly inhomogeneous network structures, characteristic of some types of real world systems, on the robustness of the power transmission grid using a complex system model of the power transmission system (OPA). It is found that as the system structure is varied from homogeneous to heterogeneous and compared to multiple unconnected small networks with the same aggregate size, the risk can undergo large changes. The unit size of the homogeneous parts of the inhomogeneous system appears to be very important in determining whether large blackouts become more likely as the system becomes more homogeneous or inhomogeneous.
\end{abstract}

\section{Introduction}

Power transmission networks and many other critical infrastructure networks come in a wide variety of shapes and sizes but overall share the characteristics that they are growing and becoming ever more critical and heavily utilized. Because of their critical importance to the functioning of modern society, it is crucial that we understand their strengths and weakness so as to understand how to reduce their risks of failure and mitigate their vulnerabilities. Different regions even within the same basic system, can have rather different characteristics and therefore it is important to understand how these regional characteristics can affect the risk for the global network. Among the characteristics that may vary from region to region are the size and degree of homogeneity of the network.

Since we are exploring the network robustness as characterized by the long-term risk of large failures and temporal dynamics, we use the OPA model. The OPA model $[1,2]$ was developed to study the long-term patterns of blackout of a power transmission system under the dynamics of an increasing power demand and the engineering responses to failure. In this model, the power demand is increased at a constant rate and is also modulated by random fluctuations. The generation capacity is automatically increased when the capacity margin is below a given critical level.

Using the OPA model we have been able to study and characterize the mechanisms behind the power tails in the distribution of the blackout size. These algebraic tails obtained in the numerical calculations are consistent with those observed in the study of the blackouts for real power systems [3, 4]. Most importantly, this model permits us to separate the underlying causes for cascading blackouts from the triggers that initiate them and therefore explore system characteristics that enhance or degrade resilience and reliability of the power transmission grid. One of these characteristics, the one investigated here, is the heterogeneity of the network.

Some real networks have an inhomogeneous structure with a series of relatively homogeneous regions coupled to each other like pearls on a string (Fig. 1). To understand the impact of inhomogeneity, specifically inhomogeneity on a certain scale, and thereby improve the realism of the model, we have investigated a new type of network structure with OPA. In this, we compare homogeneous networks to inhomogeneous networks of the type shown in figure 1 and finally compare both to a set of uncoupled small networks each with the size and structure of the 
"pearls" on the string in the inhomogeneous network. Figure 2 shows a 400 bus version of the 3 types of networks described. This will allow us to vary: 1) the number of small networks 2) the size of the small networks 3) the overall system size and therefore the scale separation between the homogeneous small parts and the overall system size 4) the total power in the different parts.

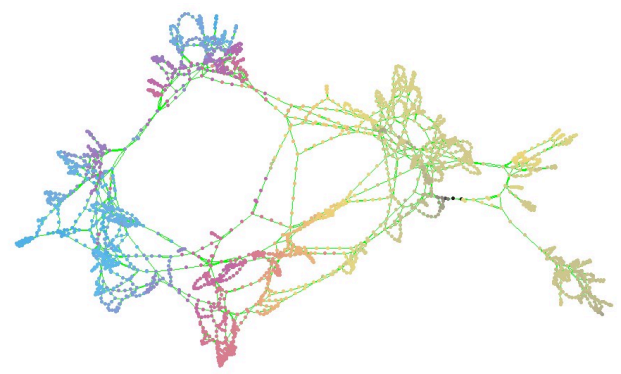

Figure $1 \mathrm{~A}$ representation of a real power transmission network

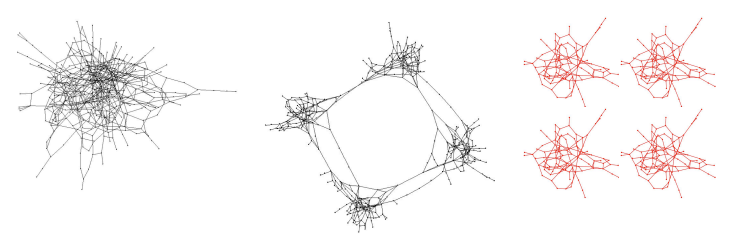

Figure 2 The types of artificial constructed networks used for this investigation. The left most panel is a homogeneous 400 bus network, the middle panel is a heterogeneous network made by connecting 4100 bus networks and the right panel is 4 unconnected 100 bus networks.

To properly evaluate the homogeneity vs. in homogeneity there must be a clear separation of scales (sizes) between the parts of the networks and the global size. This makes the problem very computationally challenging because of the long computation times to compute the complex system steady state statistics and dynamics of large networks.

The need for analysis and understanding of cascading in interconnected or coupled networks representing parts of the same or different infrastructures was recognized and described qualitatively in [5, 6] in 2003 and 2004. The effect of the coupling in such systems has been studied with several different types of models, many of which were pioneered at HICSS. In 2005, [7] gave the first analysis with coupled probabilistic cascading interconnected networks and with coupled self- organizing complex system interconnected networks. The analysis and simulation showed how the coupling could affect the critical point behavior. In 2007, [8] modeled cascading failure based on forest fire type models to study coupled networks, and in particular how the coupling affected the critical point and the power law behavior of the coupled system. In 2004, [9] applied branching processes, which have long been used to study general cascading processes, to cascading failure in power grids. In 2012, [10] used two-type branching processes to study sandpile dynamics of particles on coupled networks with the same topologies as power grids. The present paper uses self-organizing complex systems models of coupled power grids, and is the first such interacting model to use some basic power system models that reflect some power system realities in a simplified form.

In this paper, we describe the initial investigations and the impact on the long-term reliability of the system from the changes in system structure introduced in this model. Section 2 will briefly review the OPA model while section 3 will both analytically and computationally, using OPA, analyze the multiple independent unconnected small system case. Section 4 compares the multiple small unconnected systems case to the multiple connected small systems network. The $5^{\text {th }}$ section investigates the homogeneous vs. inhomogeneous case in terms of structure while section 6 discusses the case in which the additional inhomogeneity of power balance is added. Finally section 7 is a brief discussion and conclusion.

\section{The OPA model}

The OPA (ORNL-PSerc-Alaska) model for the dynamics of blackouts in power transmission systems $[1,2]$ shows how the slow opposing forces of load growth and network upgrades in response to blackouts could self organize the power system to dynamic equilibrium. Blackouts are modeled by overloads and outages of lines determined in the context of LP dispatch of a DC load flow model. This model has been found to show complex dynamical behavior [1,2] consistent with that found in the NERC data [3]. Some of this behavior has the characteristic properties of a system near a critical transition point. That is, when the system is close to a critical point, the probability distribution function (PDF) of the blackout size (load shed, customers unserved, etc) has an algebraic tail and large temporal correlation lengths are possible. One consequence of this behavior is that at these critical points, both the 
system utilization is maximized and the risk for blackouts increases sharply. Therefore, it may be natural for power transmission systems to operate close to this operating point.

The fact that, on one hand, there are critical points with maximum power served and, on the other hand, there is a self-organization process that tries to maximize efficiency and minimize risk may lead to a power transmission system that is naturally driven to this point.

In general, the operation of power transmission systems results from a complex dynamical process in which a variety of opposing forces regulate both the maximum capacity of the system components and the loadings at which they operate. These forces interact in a highly nonlinear manner and may cause a selforganization process to be ultimately responsible for the regulation of the system. This view of a power transmission system considers not only the engineering and physical aspects of the power system, but also the engineering, economic, regulatory and political responses to blackouts and increases in load power demand. A detailed, comprehensive inclusion of all these aspects of the dynamics into a single model would be extremely complicated if not intractable due to the intrinsic human interactions involved. However, it is useful to consider simplified models with some approximate overall representation of the opposing forces in order to gain some understanding of the complex dynamics in such a framework and the consequences for power system planning and operation. This is the basis for OPA.

In the OPA model the dynamics involves two intrinsic time scales. There is a slow time scale, of the order of days to years, over which load power demand slowly increases and the network is upgraded in engineering responses to blackouts. These slow opposing forces of load increase and network upgrade self organize the system to a dynamic equilibrium. There is also a fast time scale, of the order of minutes to hours, over which cascading overloads or outages may lead to blackout. The OPA model computes the long-term reliability taking into account the complex systems dynamics and feedbacks; that is, OPA is run until it converges to a complex systems steady state with stationary statistics and long time correlations. Because of the time correlations intrinsic to such a system, these simulations are different from the more common "Monte Carlo" method for generating statistics. In the case of OPA, we generally run the simulation for longer times to generate better statistics, thereby sampling more of the allowed system states with the probabilities of sampling a given state being generated by the system itself.

The main purpose of the OPA model is to study the complex behavior of the dynamics and statistics of series of blackouts in various scenarios. This allows us to easily investigate the impact of different levels of inhomogeneity on the risk and dynamics as well as other network characteristics. For the rest of the paper, OPA results are used for the computational analysis.

\section{Multiple independent networks}

To begin, we will consider a system made up of a set of $n$ separate identical small networks, each with 100 buses. We choose this system to start with as it both has analytically tractable characteristics and will be the base case that will then be used to compare with the other networks. To look at some of these characteristics, let us assume that the PDF of failure sizes of $n$ of these networks is $P_{n}(x)$ and the frequency of failures is $v_{n}$. The blackout size is measured by $\mathrm{x}$. Therefore, if we add another small network to the system, we have the probability of a failure of size $\mathrm{x}$ given by:

$$
\begin{aligned}
& v_{n+1} P_{n+1}(x)=v_{1} P_{1}(x)\left(1-v_{n}\right)+ \\
& v_{n} P_{n}(x)\left(1-v_{1}\right)+v_{1} v_{n} \int_{0}^{x} d y P_{1}(x-y) P_{n}(y)
\end{aligned}
$$

For blackouts in small networks the PDF of blackout size is very close to an exponential (this is for sizes smaller then the size at which the characteristic power law tail can start to appear, typically greater then 200 for the power grid). So we can assume:

$$
P_{1}(x)=a_{1} e^{-a_{1} x}
$$

Starting from Eq. (1) and iterating, we can find a general solution for the PDF of a system of $n$ unconnected networks has the form:

$P_{n}(x)=\frac{1}{1-\left(1-v_{1}\right)^{n}} \frac{e^{-a_{1} x}}{x} \sum_{k=1}^{n}\left(\begin{array}{l}n \\ k\end{array}\right)\left(1-v_{1}\right)^{n-k} \frac{\left(v_{1} a_{1} x\right)^{k}}{(k-1) !}(3)$

As an approximation, we assume that the variable $\mathrm{x}$ is defined between 0 and infinite. This is a good 
approximation for exponential tails. The corresponding Rank function is then:

$$
\begin{aligned}
R_{n}(x)= & \frac{e^{-a_{1} x}}{1-\left(1-v_{1}\right)^{n}}\left\{v_{1} \sum_{k=1}^{n}\left(\begin{array}{l}
n \\
k
\end{array}\right)\left(1-v_{1}\right)^{n-k} \frac{\left(v_{1} a_{1} x\right)^{k-1}}{(k-1) !}\right. \\
& \left.+\sum_{k=2}^{n}\left(\begin{array}{l}
n \\
k
\end{array}\right)\left(1-v_{1}\right)^{n-k} \frac{v_{1}^{k}}{(k-1) !} \sum_{j=1}^{k-1} \frac{(k-1) !}{(k-1-j) !}\left(a_{1} x\right)^{k-1-j}\right\}
\end{aligned}
$$

We have compared the analytical result for the Rank function, Eq. (4), with the numerical results using OPA. This comparison is shown in Fig. 3. As can be seen the fit to an exponential is reasonably good but only approximate for the $n=1$ case with a single network of 100 buses, so we cannot expect better agreement for the higher $\mathrm{n}$ results.

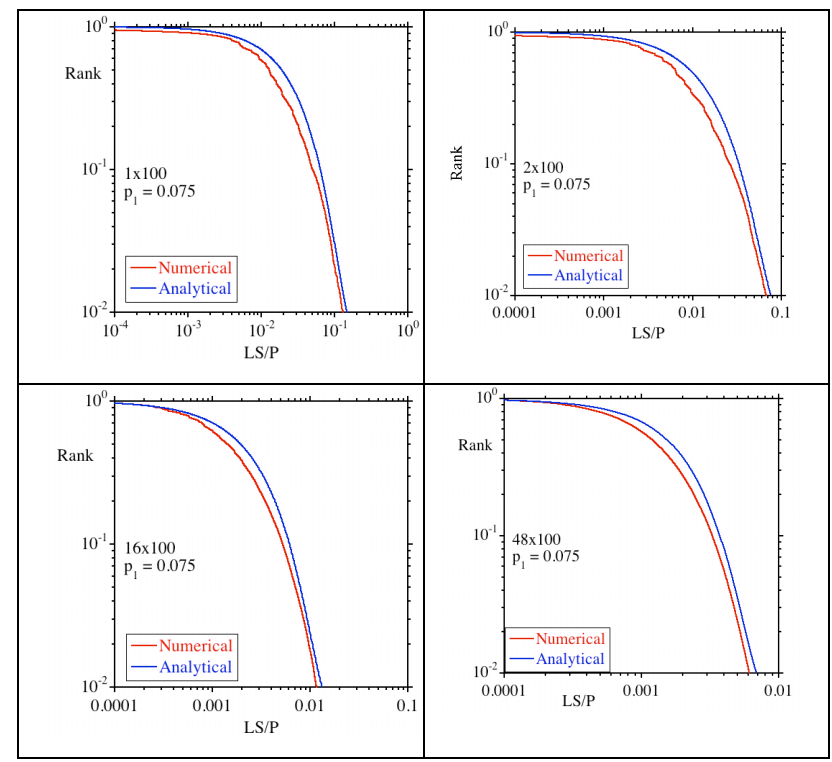

Fig. 3. Comparison of the analytical and numerical rank functions for multiple unconnected networks.

The frequency of the failures for $\mathrm{n}$ disconnected networks is given by

$$
v_{n}=1-\left(1-v_{1}\right)^{n}
$$

This solution for the frequency agrees very well with numerical results from the OPA code using multiple unconnected 100 networks as shown in Fig. 4.

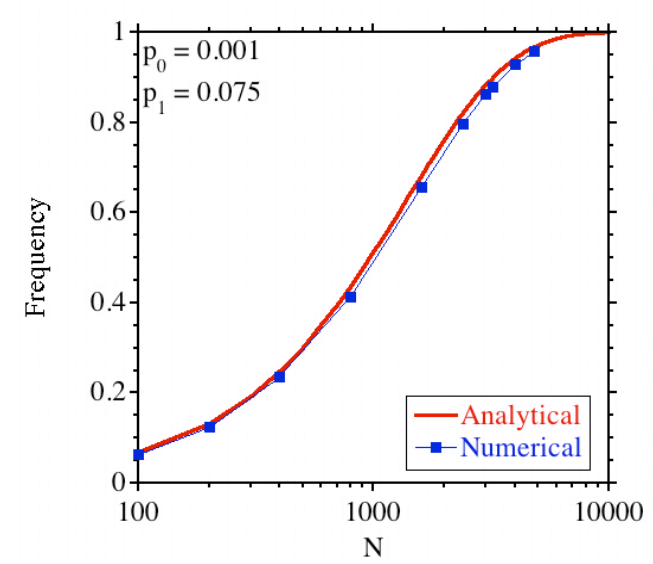

Fig. 4. Frequency of blackouts in systems of multiple 100 bus unconnected networks

These results show that small networks and by extension multiple unconnected (and therefore uncorrelated) small networks exhibit near exponential PDFs for failure size and a clearly understood frequency of failure.

\section{Multiple unconnected networks versus multiple linked networks}

In the previous section we have studied the probability of failure of a system of $n$ unconnected networks. Larger networks are a natural result of the economics/efficiency of scale. By linking these small networks (Fig. 4) it is possible to improve the operation of the system in case of local failures, for instance, a generator is down, local demand is up, or lines are outaged. In our models, the linking of the smaller homogeneous regions is done by choosing a few nodes at the edges of the network and connecting them to similar nodes in the neighboring small region. In some of these cases when the small networks are linked, power can be provided from other parts of the network to keep the whole network functioning. Once the networks are linked, we use the OPA code to dynamically evolve the system to get a reliable operating network. Thanks to the process of engineering upgrade as response to the demand, linking lines are upgraded during a transient process. Once in steady state, we then study the dynamics of the new system by running normally for the equivalent of 105 years. In this way, we collect enough information for the statistical studies. By linking small networks, we improve the efficiency of the overall system by reducing the frequency of failures. This is shown in Fig. 5, where we have plotted the frequency of blackouts as a function of the overall size of the network for three different network 
configurations: 1) n multiple unconnected 100-bus networks, 2) a homogeneous $n \times 100$ bus network and 3) n 100-bus networks linked by a few lines. Figure 4 is an example of this last type configuration for $n=8$. The parameters used in these calculations are $\mathrm{p}_{0}=$ 0.00025 and $\mathrm{p}_{1}=0.037$. It can be clearly seen in figure 5 that the connected networks, both the linked 100 bus system and the homogeneous network do have a significant improvement in the frequency of failures due to the connected nature of the grid.

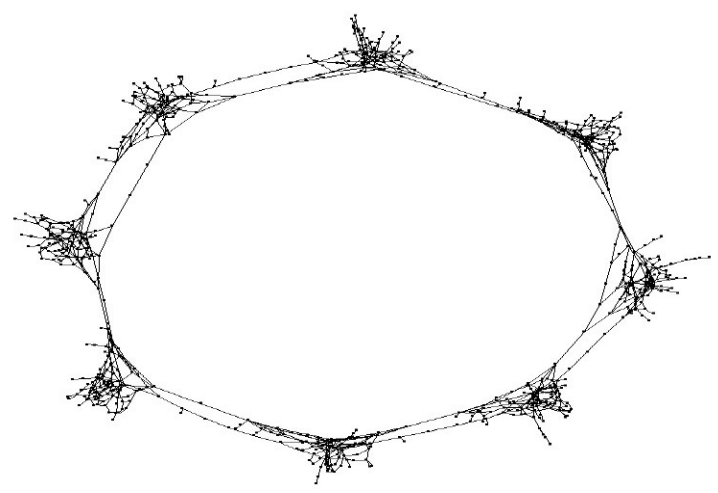

Fig. 4. An eight 100 bus linked network

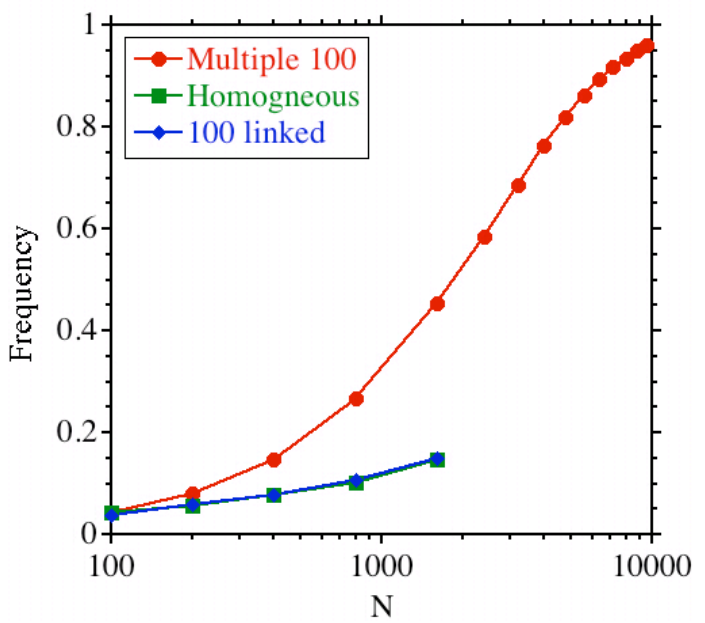

Fig. 5. Frequency of blackouts as a function of the overall size of the network

However, although the frequency of blackouts decreases when we link the networks, not all characteristics are positive for the operation of the fully connected system. When we link networks in order to improve efficiency and decrease the frequency of the blackouts, we also increase the risk of very large blackouts. This is shown in Fig. 6 where we have plotted the rank function (that is, the complementary CDF) of the blackout size for two 1600 bus network, one with 16100 bus unconnected networks and the other with 16100 bus linked networks. From figure 5 one can see there is almost a factor of 3 decrease in the frequency of failures between the linked and unlinked 1600 bus systems, but figure 6 clearly shows that though there is a reduction in probability of the small events, there is a large increase in the probability of the large events with the CCDF no longer being well fit with an exponential.

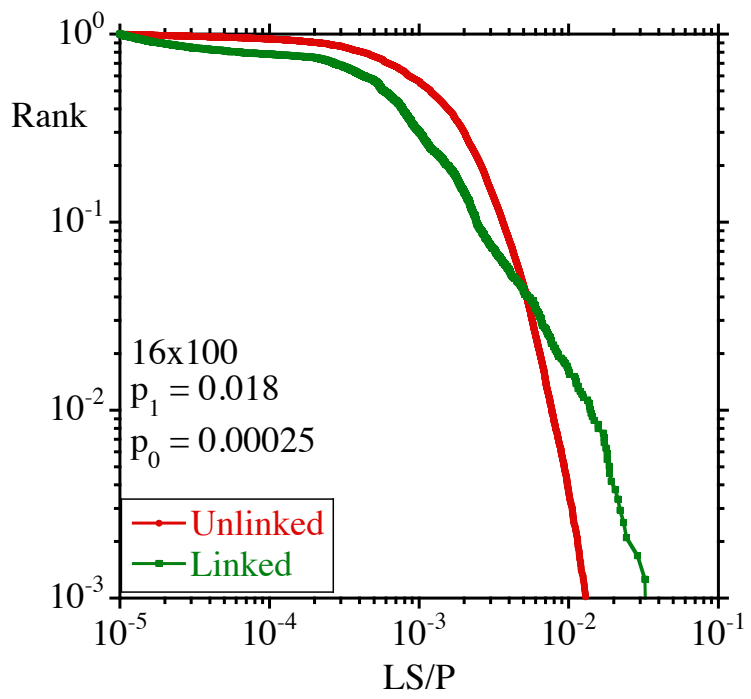

Fig. 6. Rank function of the blackout size for two 1600 bus networks. The red curve is for 16 unlinked 100 bus networks and the blue is for 16 linked 100 bus networks.

We will call the small networks that are used to make up the large system zones so as to be able to investigate the differences between the connected and unconnected systems. To understand the cause of the increase probability of the large blackouts, we can look at what happens in the different zones when there is a blackout. We see that the number of zones involved in a blackout decreases exponentially in the case of multiple non-connected systems. This is consistent with the individual units being unlinked uncorrelated systems with their own random drive, and it arises from the rare possibility that two or more blackouts happen independently in the different zones. In particular, the exponential dependence for larger blackouts arises from multiplying the probabilities of the independent zone blackouts. However, in the case of the linked system, the 
probability of several zones being involved in a blackout increases significantly, as can be seen in Fig. 7. The deviation of the probability from the unlinked system is most pronounced with the larger number of zones, suggesting quite reasonably that the largest correlations across the linked zones occur when the failures are largest, forcing the propagation across the links connecting the individual zones. Similar behavior has been found when looking at the synchronization function in coupled infrastructures [5]. It is worth noting that in this initial work, the failure probabilities and upgrade rules are the same for the linking lines as they are in the clusters. Modifications to this are being investigated as it is likely more realistic to have different failure probabilities and upgrade rates for the connecting lines then for the internal lines in the zones.

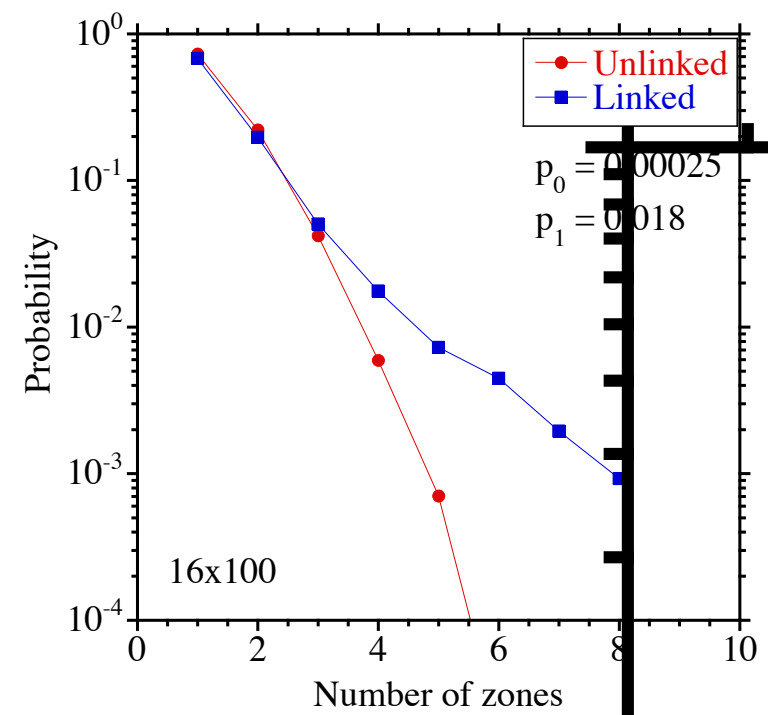

Fig. 7. Probability of a blackout occurring across a "Number of zones" at the same time.

\section{Homogeneity versus Inhomogeneity}

The differences between the multiple unconnected, independent zones and the linked zones, is what might be expected. However, a more interesting question is what happens when one compares the linked zones to a homogeneous system with the same number of buses. These are among the differences found in the networks for different regions (Western US vs Eastern US for example). There are considerable obvious differences between the networks obtained by linking small networks, as shown in Fig. 4 and a homogeneous network with the same number of buses as shown in Fig. 8.

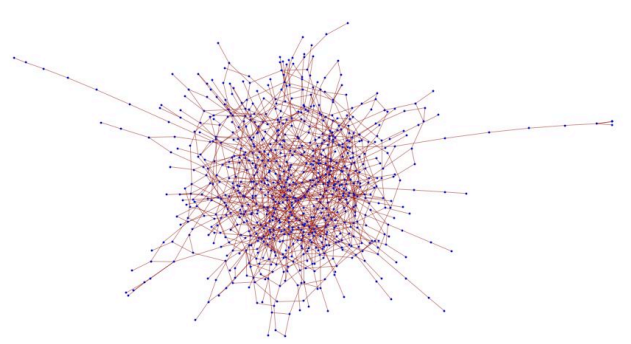

Fig. 8. Homogeneous 800 bus network

Their characteristics are also quite different. The homogeneous large networks have rank functions for the blackout size that are characterized by a clear power tail. The situation for the linked networks of the same sizes is less clear. The tails show higher probability for the largest blackout but the power tail is less obvious, as can be seen in Fig. 9 for networks with 1600 buses. The change appears as a higher tail or less steep power law, consistent with the results seen as a function of the number of zones involved from fig. 7. This might be an issue with the size of the individual small systems that make up the linked network.

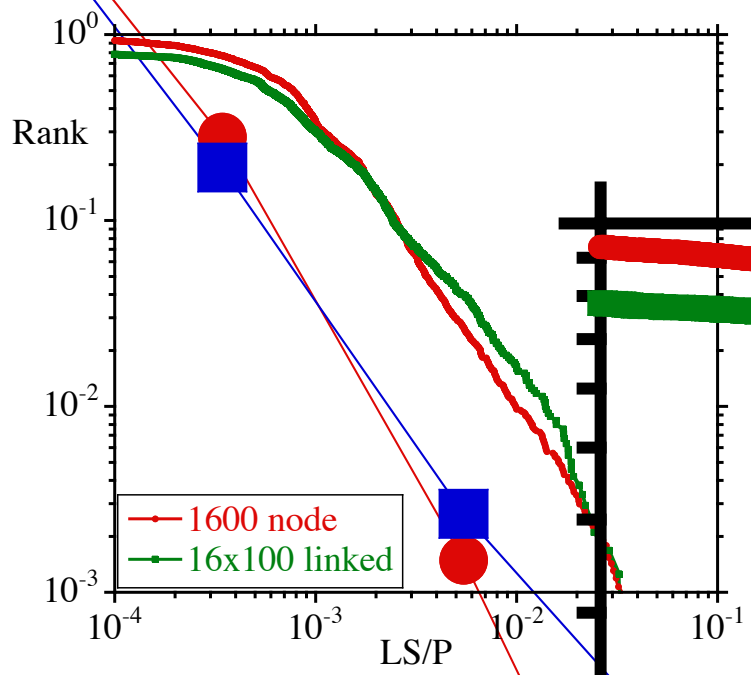

Fig. 9. Rank function of the blackout size for $\mathrm{p} 0=0.00025$ and $\mathrm{p} 1=0.018$.

In spite of the less clear power tail, we can always fit the tail of the rank function and use the exponent as a relative measure of how fast the tail falls off. The exponent of the rank function for the linked networks is in general lower than the exponent for the same 
size homogeneous network. That implies that the risk of large blackouts is higher for the linked multiple networks. This difference between the exponents of the different systems increases as the reliability of the system, $\mathrm{p}_{1}$, decreases. This is shown in Fig. 10 for the networks made up by linked 100 bus networks as compared with the homogeneous networks. This again is consistent with the bump on the tail coming from the increased probability of large failures propagating across the links. As $\mathrm{p}_{1}$ is the probability of propagation, with small $\mathrm{p}_{1}$, only the largest events have a high probability of propagating across the links but with higher $\mathrm{p}_{1}$, smaller events can propagate across the links, making the system act more like a larger homogeneous system. However, when we consider linked 200 bus or larger networks then the heavier tails in the individual "little" systems makes a larger event more probable and therefore increases the probability of a failure propagating across the link. In addition the scale separation between the components and overall network might not be large enough to see differences.

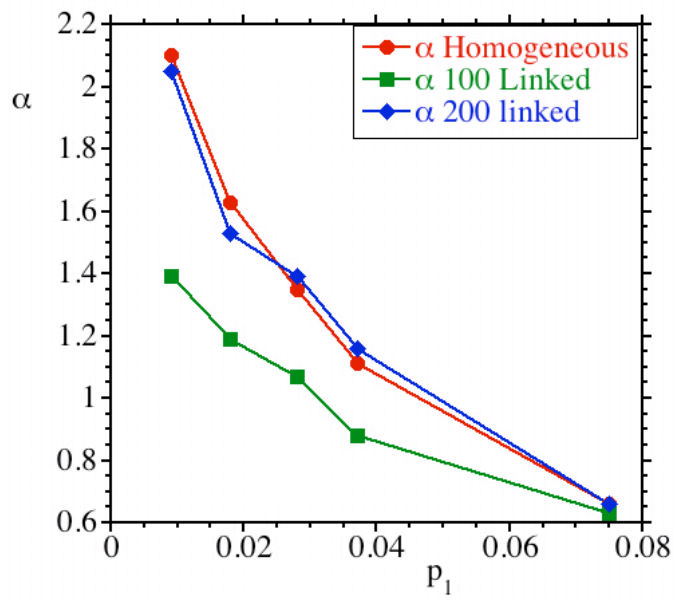

Fig. 10. Exponent of tail of CCDF as a function of $p_{1}$, the reliability parameter for homogeneous systems and linked systems.

\section{Linking networks with the different power levels}

In the linked networks we have looked at so far, each of the small grid zones have had the same total load and generation mix within them, ie they were individually capable of generating their needed power in the unperturbed state and that needed power was the same for all the small regions. It is therefore the fluctuations in the load in the individual regions that force the flow of power across the links. However, in the real world the generation power and loads in the small zones are not all the same which in turn can cause much larger unbalanced flows across the links. Indeed, transfers of power between zones yields important economic and environmental benefits. We therefore investigate the impact of variation in total power in a zone of the inhomogeneous network. In this study, we choose one of the 100-bus small networks in the linked system and we multiply its generator power and loads by $\mathrm{a}$ factor $\mathrm{k}$ before linking it to the rest of the network. For simplicity we consider the case of a 4 100-bus linked network. To the modified 100-bus component we assign the zone number 1 . If $\mathrm{k}>1$, the loads and generators of zone 1 have levels higher than the others (ie it is a power exporting region), if $\mathrm{k}$ $<1$, we have the reversed situation (a power importing region). In the present study, we have varied $\mathrm{k}$ from 0.25 to 8 by factors of 2 .

In Fig. 11, we have plotted the frequency of the blackouts and the averaged normalized load shed as a function of the factor $\mathrm{k}$. We can see that the frequency of the blackouts decreases as $\mathrm{k}$ increase, while the averaged normalized load shed increases. The changes are small, but they seem to be systematic. This is consistent with the results from the previous section. With a larger $\mathrm{k}(\mathrm{k}>1)$, larger flows can be forced across the links by the load fluctuations in the region, making the system more strongly connected and therefore more likely to have large failures propagate across the links. Conversely, when $\mathrm{k}$ is small (less then 1) the connections are effectively weakened because less flow is forced across the links, making the large failures less likely and making the system look more like the uncoupled system with a higher frequency of failure but fewer large failures.

In this case, it is interesting to see what is happening in each of the four zones. In Fig. 12, we have plotted the averaged ratio of the load demand to the generation power in each zone. We can see from the figure that on average the zones are well balanced. As indicated before, the OPA evolution during the transient phase produces effective upgrades of the connecting lines in such a way that the system is reliable. In the different zones, the generation margin stays close to 0.20 . No obvious unbalances are shown. 


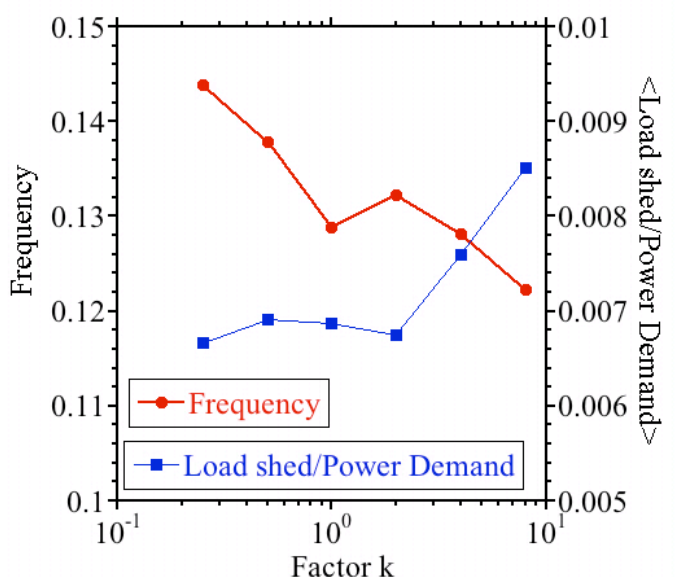

Fig. 11. Frequency and normalized load shed as a function of the imbalance of the power in the small pieces of the linked network.

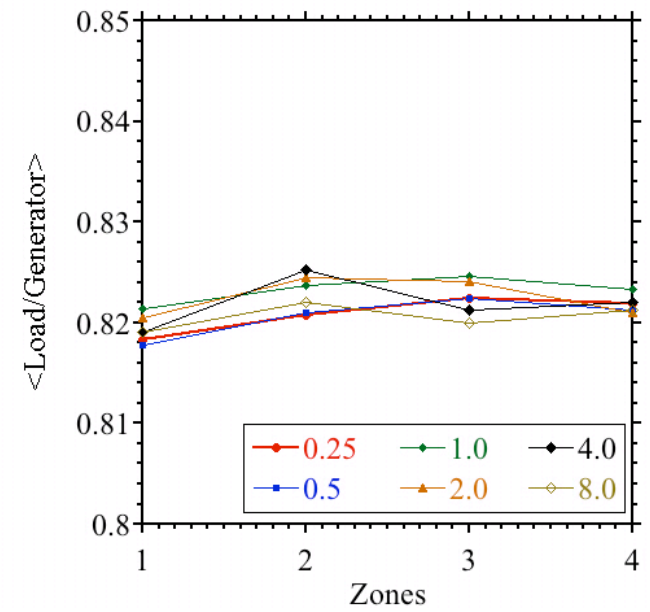

Fig. 12. Average load to generation ratio in each zone.

However, if we look at the load shed in each zone, we can see that the averaged load shed is larger in the zone or zones that import power. This is shown in Fig. 13, where we have plotted the averaged normalized load shed as a function of zone number for all the values of the factor $k$ that we have considered. The result is systematic and can be understood as coming from the inability of that zone being able to supply the needs of it's power exporting neighboring zones when they have a large load fluctuation.

When $\mathrm{k}<1$, only zone one imports power, there is an increase of the load shed in this zone but since zone one is only one zone of the four, its overall impact is small. However, for $\mathrm{k}>1$, there are three zones with lower generation. The averaged load shed increases in the three zones and in this case has a stronger impact on the overall load shed. This causes the stronger coupling across the entire system and explains the dependence of the global averaged load shed as a function of $\mathrm{k}$ shown in Fig. 11.

The average fractional line loading, $\langle\mathrm{M}>$, in each region behaves similarly to the load shed. For $\mathrm{k}>1$, the $\langle\mathrm{M}>$ in zone 1 is lower than in the other zones and the reverse occurs for $\mathrm{k}<1$ as it is shown in Fig. 14. The zones with less generation power are more stressed than the others due to the large fluctuations in the demand from the higher power neighboring regions. These large fluctuations once again increase the effective coupling.

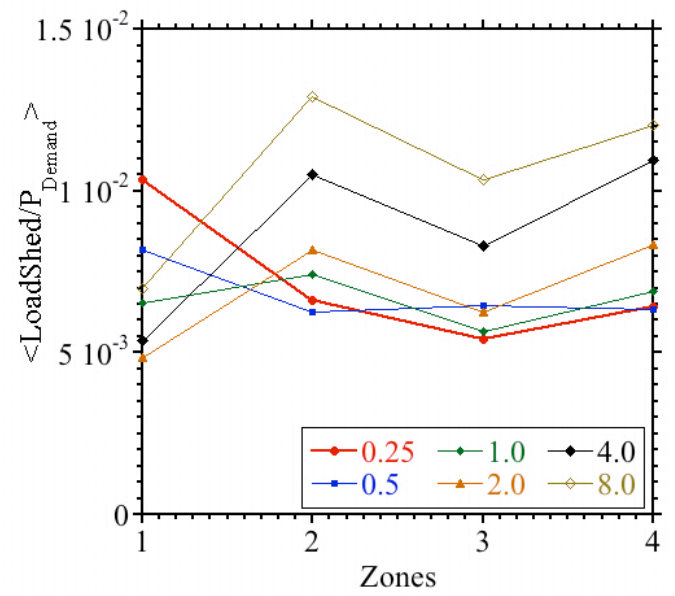

Fig. 13 Normalized average load shed in each zone.

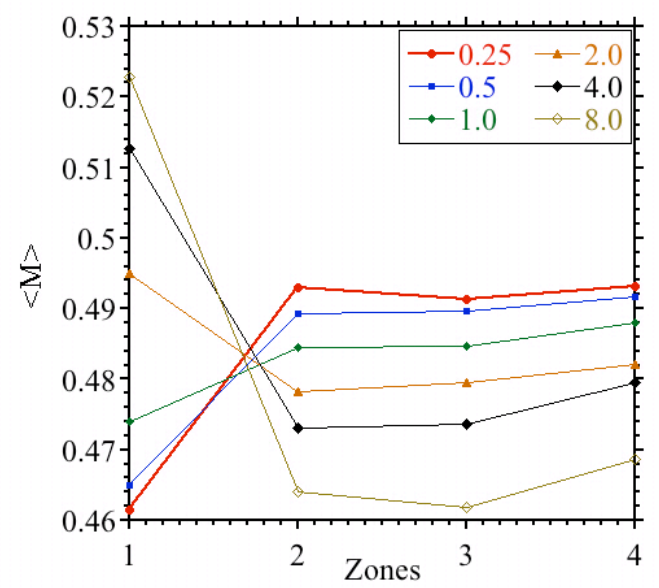

Fig. 14 The fractional average line loading for the different zones for different values of the power imbalance factor $k$. 


\section{Discussion and conclusions}

Using a complex systems power transmission grid model (OPA) to investigate the impact of grid inhomogeneity on the system, preliminary analysis suggests that in some cases the highly inhomogeneous network can have a higher risk of large failures than both the uncoupled individual systems and a homogeneous grid of comparable size. Additional inhomogeneities in the form of imbalances in the power in the different zones in the system added to the risk in the system. A likely mechanism for this is the failure size dependent effective coupling between the different zones. It is important to note that these results might change as the size of the individual parts get larger and move to a size which exhibits a heavy tail in the individual regions. It should also be noted that the rules for power dispatch are system-wide in our present implementation and if dispatch were weighted toward the local zone, that too could have an impact on the results.

The next step in this investigation is to vary the size of the small homogeneous regions (the zones) to find if these results change as these regions grow to the point that they have heavy tails of their own. Then, the power balance issues will be further explored to include a real load/generation imbalance causing systematic long-distance power transfers such that one region (zone) is not locally in balance and imports power from other regions in the base case operation. Then the dispatch rules will be changed to investigate the impact of local versus system-wide power dispatch. And finally, islanding schemes can be investigated to see the impact of cutting the zones apart on the spread of the failures.

It is clear that inhomogeneities can have a number of impacts on the system robustness and reliability coming from both the inhomogeneity in the network structure and in the balance of power across the inhomogeneous network. These seem to work together to move the system closer to or perhaps even past its critical point leading to a heavy tail or even a bump on the tail blackout (failure) size distribution with the increased large blackout risk inherent to those distributions.

While the work presented here is specifically for a single type of infrastructure (the power transmission grid as modeled by OPA) that has an inhomogeneous structure, similar results likely would hold for coupled infrastructures where the inhomogeneity comes in the coupling.

\section{Acknowledgements}

We gratefully acknowledge funding provided in part by the California Energy Commission, Public Interest Energy Research Program. This paper does not necessarily represent the views of the Energy Commission, its employees or the State of California. It has not been approved or disapproved by the Energy Commission nor has the Energy Commission passed upon the accuracy or adequacy of the information. DEN thanks the financial support of Universidad Carlos III and Banco Santander through a Càtedra de Excelencia. ID gratefully acknowledges support in part of this work by NSF grant CPS1135825 .

\section{References}

[1] B. A. Carreras, V.E. Lynch, I. Dobson, D.E. Newman, Chaos 14, 643 (2004).

[2] I. Dobson, B.A. Carreras, V.E. Lynch, D.E. Newman Chaos: An Interdisciplinary Journal of Nonlinear Science, vol. 17, no. 2, June 2007.

[3] B. A. Carreras, D. E. Newman, I. Dobson, and A. B. Poole, IEEE Transactions on Circuits and Systems I-regular papers, 51, 1733 (2004).

[4] Xingyong Zhao, Xiubin Zhang, and Bin He, Energy conservation and Management, (2008).

[5] R. G. Little, Toward More Robust Infrastructure: Observations on Improving the Resilience and Reliability of Critical Systems, 36th Annual Hawaii International Conference on System Sciences, HI 2003.

[6] S. M. Rinaldi, Modeling and Simulating Critical Infrastructures and Their Interdependencies, 37th Hawaii International Conference on System Sciences, Big Island, HI, USA, January 2004.

[7] D. E. Newman, B. Nkei, B. A. Carreras, I. Dobson, V. E. Lynch, P. Gradney, Risk assessment in complex interacting infrastructure systems, 38th Hawaii International Conference on System Sciences, Hawaii, January 2005.

[8] B.A. Carreras, D.E. Newman, P. Gradney, V.E. Lynch, I. Dobson, Interdependent risk in interacting infrastructure systems, 40th Hawaii International Conference on System Sciences, Hawaii, January 2007.

[9] I. Dobson, B.A. Carreras, D.E. Newman, A branching process approximation to cascading load-dependent system failure, 37th Hawaii International Conference on System Sciences, Hawaii, 2004.

[10] C. D. Brummitt, R. M. D’Souza, and E. A. Leicht. Suppressing cascades of load in interdependent networks, Proceedings of the National Academy of Sciences 109.12 (2012): E680-E689. 